\title{
Euphorbia kansui Attenuates Insulin Resistance in Obese Human Subjects and High-Fat Diet-Induced Obese Mice
}

\author{
Seung-Wook Lee, ${ }^{1}$ Hyun-Young Na, ${ }^{1}$ Mi Hyeon Seol, ${ }^{1}$ Mia Kim, ${ }^{2}$ and Byung-Cheol Lee ${ }^{1}$ \\ ${ }^{1}$ Department of Clinical Korean Medicine, Graduate School, Kyung Hee University, 26 Kyungheedae-ro, Dongdaemun-gu, \\ Seoul 02447, Republic of Korea \\ ${ }^{2}$ Department of Cardiovascular and Neurologic Disease (Stroke Center), College of Korean Medicine, Kyung Hee University, \\ 23 Kyungheedae-ro, Dongdaemun-gu, Seoul 02447, Republic of Korea \\ Correspondence should be addressed to Byung-Cheol Lee; hydrolee@khu.ac.kr
}

Received 13 May 2017; Revised 20 July 2017; Accepted 27 August 2017; Published 4 October 2017

Academic Editor: Elzbieta Janda

Copyright (C) 2017 Seung-Wook Lee et al. This is an open access article distributed under the Creative Commons Attribution License, which permits unrestricted use, distribution, and reproduction in any medium, provided the original work is properly cited.

\begin{abstract}
Background. Obesity is a main cause of insulin resistance (IR), metabolic syndrome, and fatty liver diseases. This study evaluated Euphorbia kansui radix (Euphorbia) as a potential treatment option for obesity and obesity-induced IR in obese human and high-fat diet- (HFD-) induced obese mice. Methods. In the human study, we analyzed the body weight change of 14 patients who took a single dose of $6 \mathrm{~g}$ of Euphorbia powder. In the animal study, male mice were divided into three groups: normal chow, HFD, and Euphorbia (high-fat diet and $100 \mathrm{mg} / \mathrm{Kg}$ Euphorbia once per week). Body weight, epididymal fat pad weight, fasting blood glucose, fasting insulin, HOMA-IR, and oral glucose tolerance test were measured. Also, macrophage infiltration and expression of CD68, tumor necrosis factor- (TNF-) $\alpha$, interferon- (IFN-) $\gamma$, and interleukin- (IL-) 6 genes in the liver and adipose tissue were analyzed. Results. The human study showed that Euphorbia has a potential effect on body weight loss. In the in vivo study, body weight, epididymal fat weight, glucose level, IR, expression of CD68, TNF- $\alpha$, IFN-r, and IL-6 genes, and macrophages in liver and adipose tissue were significantly reduced by Euphorbia. Conclusions. These results suggest that Euphorbia attenuates obesity and insulin resistance via anti-inflammatory effects.
\end{abstract}

\section{Introduction}

Obesity is rapidly increasing worldwide [1]. Increased visceral adipose tissue confers a proinflammatory milieu and insulin resistance (IR) [2], which is directly or indirectly related to various health problems including type 2 diabetes mellitus (T2DM), hypertension, dyslipidemia, and fatty liver diseases, resulting in an annual cost of about 150 billion dollars [3].

Many efforts, including lifestyle changes and antiobesity drugs, have been applied for obesity treatment. However, lifestyle change is not ensured to maintain weight loss for more than a few years, and the use of drugs has been limited by side effects such as negative mood changes, suicidal thoughts, and gastrointestinal or cardiovascular complications $[4,5]$. Therefore, new efforts, including herbal medicines, can provide an alternative therapy for this medical challenge.
The root of Euphorbia kansui Liou (Euphorbia) is an herb that has been commonly used in China for thousands of years. It is in the cathartic (resolving water retention through stool) category of the Chinese medical literature [6]. In clinics, it is often used to treat ascites caused by hepatocirrhosis [7], pancreatitis [8,9], and intestinal obstruction [10]. In recent years, there have been reports of its antitumor [11] and antiviral role $[12,13]$ as well as its ability to regulate the immune system [14]. Moreover, recent studies have revealed that Euphorbia has anti-inflammatory [15], antidiabetic [16], and antiobesity effects [17].

In this study, we evaluated the effects of Euphorbia on obesity, glycemic control, and IR. In addition, we addressed the safety issue of Euphorbia and investigated its mechanisms of action, especially with respect to the inflammatory reaction and macrophage infiltration in liver and adipose tissue. 


\section{Materials and Methods}

2.1. Preparation of Euphorbia. Euphorbia was purchased from the Department of Pharmaceutical Preparation of Kyung Hee University Oriental Medical Hospital (Seoul, South Korea). The original source of the Euphorbia was Kyung Hee Herb Pharm (WonJu, South Korea). Drug quality was tested based on the standards of the Korea Food and Drug Administration and the standards of the hospital. Extractions were prepared from dried Euphorbia. Euphorbia (100 g) was added to $1,500 \mathrm{ml} 80 \%$ ethanol and boiled for 2 hours using a heating mantle. The sieve-filtered solution was filtered into a $500 \mathrm{ml}$ flask and concentrated with a rotary evaporator (model NE-1, EYELA Co., Tokyo, Japan). The solution was freeze-dried, and the Euphorbia extract was stored at room temperature. The final collection yield was $9.6 \%$.

2.2. Human Study. The inclusion criteria were as follows: BMI over 23, age between 19 and 65 years, ability to provide informed consent, and stable health according to the opinion of the investigator. The exclusion criteria included pregnant or lactating women, participants who had undergone another obesity treatment in the previous 3 months, participants of clinical trials within the previous 3 months, clinically significant new illness in the 1 month before screening, or not being suitable for participating in the study in the opinion of the investigator including an existing physical or mental condition, significant change in smoking habits within the previous 3 months, and diseases including epilepsy, uncontrolled hypertension, or hypotension, cerebrovascular diseases, and clinically significant abnormal hepatic (e.g., AST or ALT greater than $2.5 \times$ ULN or total bilirubin greater than $1.5 \times \mathrm{ULN}$ ) or renal function lab tests (e.g., creatinine greater than $1.25 \times \mathrm{ULN})$, suggestive of hepatic or renal impairment.

Eligible patients recruited from January 2014 to October 2014 were given a single $6 \mathrm{~g}$ dose of raw Euphorbia powder with simple dietary consultations at the Korean Medicine Hospital of Kyung Hee University, Seoul, South Korea. The consultations include taking 3 meals a day regularly and prohibiting confections. Patients were analyzed for body weight (BW), height, waist-hip ratio, body fat percentage, aspartic aminotransferase (AST), alanine aminotransferase (ALT), gamma glutamyltranspeptidase (GGT), blood urea nitrogen (BUN), and creatinine before and one month after treatment. Body mass index (BMI) and CockCroft-Gault estimated glomerular filtration rate (eGFR) were calculated from the above data. Furthermore, other side effects were noted after Euphorbia administration. BW, BMI, waist-hip ratio, and body fat percentage of patients were estimated using a body composition analyzer (Inbody Co., Seoul, Korea). This study was approved by the International Review Board of Kyung Hee Hospital (KOMCIRB-2014-06).

2.3. Animals and Diets. The animals used in this study were 19-21 g, 5-week-old, male C57BL/6 mice (Central LabAnimals, Inc., Korea). To determine the optimal dose of Euphorbia, thirty-five mice fed normal chow (NC) with $10 \%$ fat were randomized to be treated with the following dose levels of Euphorbia with five mice per dose level: no treatment, $20 \mathrm{mg} / \mathrm{kg}, 50 \mathrm{mg} / \mathrm{kg}, 100 \mathrm{mg} / \mathrm{kg}, 200 \mathrm{mg} / \mathrm{kg}, 500 \mathrm{mg} / \mathrm{kg}$, and $1000 \mathrm{mg} / \mathrm{kg}$ of Euphorbia were orally administered once weekly for 28 days. Upon completion of the study, all mice were weighed and sacrificed, and the liver, spleen, and kidney were harvested and weighed. After 28 days of treatment, an $18.2 \%$ reduction of body weight was achieved with the $100 \mathrm{mg} / \mathrm{kg}$ dose level compared to the no treatment control group. No differences of liver, spleen, and kidney weight were observed with $100 \mathrm{mg} / \mathrm{kg}$ dose Euphorbia, finally, the $100 \mathrm{mg} / \mathrm{Kg}$ of Euphorbia was selected for main experiment. With free access to water and food, the mice were fed a highfat diet (HFD) with $60 \%$ fat for 13 weeks in order to induce obesity, except in the group NC with $10 \%$ fat. After 8 weeks of the HFD diet, the HFD groups showed significant body weight differences compared to the NC-fed group (28.98 \pm $1.40 \mathrm{~g}$ and $41.26 \pm 1.14 \mathrm{~g}$, resp., $p<0.001)$. The HFDfed mice were randomly assigned to two groups: HFD and Euphorbia groups ( $n=5$ in each). For the remaining 5 weeks of the experiment, the Euphorbia group was fed Euphorbia $(100 \mathrm{mg} / \mathrm{kg}$ ) extract once a week, while the NC and HFD groups were administered normal saline. All experiments were carried out in accordance with guidelines of the Korean National Institute of Health Animal Facility. The Animal Care Committee at Kyung Hee University approved all protocols used in this study (KHMC-IACUC 14-025).

2.4. Body and Epididymal Weight Measurement. Body weight (BW) was recorded at the beginning and end of the experiment using an electronic scale (CAS 2.5D, Seoul, Korea) and was measured at the same time in the morning every day. The mice were killed at 13 weeks after blood collection by heart puncture. The epididymides and livers were removed rapidly and weighed.

2.5. Insulin Concentration and Insulin Resistance Measurement. At the 11th week of the experimental period, we withdrew blood from the tail vein of mice after 6 hours of fasting. Serum insulin concentration was measured using an ultrasensitive mouse insulin ELISA kit (Crystal Chem Inc., USA). Insulin resistance was calculated using the following equation: HOMA-IR = fasting blood glucose $(\mathrm{mg} / \mathrm{dl}) \times$ fasting blood insulin $(\mathrm{mg} / \mathrm{ml}) \times 0.0717225161669606$.

2.6. Oral Glucose Tolerance Test (OGTT). At the 12th week, an oral glucose tolerance test was performed after the mice had undergone 14 hours of fasting. The fasting (baseline) blood glucose measurement was performed by applying a drop of tail blood to a strip-operated blood glucose sensor (ACCU-CHECK Performa, Australia). Glucose ( $2 \mathrm{~g} / \mathrm{kg}$ body weight) was administered orally to each animal. Tail vein blood samples were withdrawn at 30, 60, and 120 minutes after glucose administration.

2.7. Stromal Vascular Cell (SVC) Segregation. Epididymal fat pad tissue, obtained at week 13 of the experimental period, was mixed with a solution of phosphate-buffered saline (PBS, Gibco, USA) and 2\% bovine serum albumin (BSA, Gibco, USA). After cutting the fat pad into $1 \sim 2 \mathrm{~mm}$ sized round 
shapes with scissors, collagenase (Sigma, USA) and DNase I (Roche, USA) were added. A $100 \mu \mathrm{m}$ cell strainer (BD Biosciences, USA) was used to remove extraneous tissue.

2.8. Fluorescence Activated Cell Sorting (FACS) Analysis of Adipose Tissue Macrophages (ATMs). The number of SVCs obtained from the adipose tissue was counted by cellometer (Nexcelom Bioscience LLC, USA), and every sample was set at a concentration of $10^{6}$ cells. FcBlock (BD Pharmingen, USA) was mixed with the sample at a ratio of $1: 100$, and the reaction was performed for 10 minutes. Fluorophoreconjugated antibodies were added to the shaded state and reacted for 20 minutes. The following antibodies were used: CD45-APC Cy7 (Biolegend, USA), CD68-APC (Biolegend, USA), CD11c-phycoerythrin (CD11b-PE, Biolegend, USA), and CD206-FITC (Biolegend, USA). The samples were analyzed by FACSCalibur (BD Biosciences, USA). A FlowJo (Tree Star, Inc., USA) was used to analyze the percentage of macrophages.

2.9. Immunofluorescence Staining of Stromal Vascular Cells. SVCs were plated onto glass coverslips for 2 hours, and nonadherent cells were removed by washing in PBS. The cells were fixed in $10 \%$ formalin for 20 minutes and then blocked in 2\% BSA in PBS. Lipid staining was performed by incubation of fixed cells in $1 \mathrm{ug} / \mathrm{ml}$ Bodipy (Sigma-Aldrich) in PBS for 5 minutes. Coverslips were mounted on slides with Vectashield (Vector Laboratories, Burlingame, CA) and imaged under fluorescence microscopy (Olympus BX51, Olympus, Japan).

2.10. Immunohistochemistry. Immunohistochemistry was performed on $10 \%$ buffered formalin-fixed, paraffin-embedded liver tissues using antibodies against F4/80 (1:100, Genetex, Irvine, CA), followed by incubating in biotinylated antimouse/rabbit/goat IgG $(\mathrm{H}+\mathrm{L})$, made in horse (Vector Laboratories, Inc.). Liver tissue sections were consecutively stained with avidin-biotin horseradish peroxidase complex (Vectastain ABC ELITE kit; Vector Laboratories, Burlingame, CA) for $15 \mathrm{~min}$ before a substrate solution of $3,3^{\prime}$-diaminobenzidine tetrahydrochloride (DAB; Sigma Chemical, St. Louis, MO) was added. The sections were photographed under an Olympus photomicroscope (Olympus BX-50, Olympus Optical, Tokyo, Japan). The percentage of F4/80positive area in liver tissues was analyzed using the ImageJ software (NIH, USA).

2.11. RNA Extraction and Real-Time Reverse TranscriptasePolymerase Chain Reaction. Mini RNA Isolation IITM (ZYMO RESEARCH, CA, USA) was used for separation of RNA from liver and epididymal fat pads. To evaluate the gene expression of CD68 (macrophage marker), tumor necrosis factor- $\alpha$ (TNF- $\alpha)$, interferon- $\gamma$ (IFN- $\gamma$ ), and interleukin6 (IL-6) in adipose tissue, we performed quantitative reverse transcriptase-polymerase chain reaction (qRT-PCR). Primers for the analysis were designed as follows: CD68,
$5^{\prime}$-TTCTGCTGTGGAAATGCAAG and $5^{\prime}$-AGAGGGGCTGGTAGGTTGAT; TNF- $\alpha, 5^{\prime}$-TTCTGTCTACTGAACTTCGGGGTGATCGGTCC and $5^{\prime}$-GTATGAGATAGCAAATCGGCTGACGGTGTGGG; IFN- $\gamma, 5^{\prime}$-ACTGGCAAAAGGATGGTGAC and $5^{\prime}$-TGAGCTCATTGAATGCTTGG; IL-6, 5'-AACGATGATGCACTTGCAGA and 5'-GAGCATTGGAAATTGGGGTA; GAPDH, which was used as a housekeeping gene, $5^{\prime}$-AGTCCATGCCATCACTGCCACC and 5 -CCAGTGAGCTTCCCGTTCAGC. The threshold cycle (TC) of each gene expression, determined by SDS Software 2.4 (Applied Biosystems ${ }^{\circledR}$, USA), was converted into Relative Quantitation (RQ) based on GAPDH, and the calculated fold change value was used for gene expression analysis. The fold change value of the experimental group was converted based on the NC group value, which was considered to be one.

2.12. Statistical Analysis. All calculations were performed using GraphPad PRISM 5 (GraphPad Software, Inc., San Diego, CA, USA). All values are expressed as mean \pm SE. The significance of differences between groups was determined using one-way analysis of variance (ANOVA), followed by Tuckey's post hoc test. All $p$ values were two-tailed, and significance was set at $p<0.05$.

\section{Results}

3.1. Effects of Euphorbia in Overweight or Obese Human Patients. The BW changes of 14 patients were obtained at one month after one-time treatment with $6 \mathrm{~g}$ Euphorbia. There was significant decrease in BW and BMI after Euphorbia powder administration $(65.37 \pm 3.53 \mathrm{~kg}$ versus $64.10 \pm 3.49 \mathrm{~kg}$ for BW and $24.93 \pm 0.65$ versus $24.28 \pm 0.58$ for BMI, $p<$ 0.05 ) (Table 1). There was no significant difference in BUN, creatinine, eGFR, AST, ALT, GGT, protein, albumin, fasting glucose, and HbAlc before and after administration with Euphorbia (Table 1). As Euphorbia is traditionally classified as a cathartic herb, patients underwent an average of $0.92 \pm 0.27$ cases of vomiting and $8.08 \pm 1.24$ cases of diarrhea. There were several mild side effects including 1 report of mild abdominal pain and 6 cases of abdominal discomfort, which diminished after a few days.

\subsection{Effects of Euphorbia on BW and Epididymal Fat Weight} Changes in Mice. The fasting body weight (FBW) of the HFD group increased significantly compared to that of the NC group ( $p<0.05$ ), and Euphorbia group showed a decrease in BW compared to the HFD group $(p<0.05)$ (Figure 1(a)). The weight of epididymal fat also was significantly increased in the HFD group compared to the NC and was decreased by Euphorbia $(p<0.001)$ (Figure 1(a)).

3.3. Effects on Blood Glucose and Insulin Resistance in Mice. Fasting blood glucose (FBG) was significantly higher in the HFD group than NC group ( $p<0.001)$, and it was significantly lower in the Euphorbia group than HFD group $(p<$ $0.01)$. Fasting insulin concentration (FI) was significantly higher in the HFD group compared to the NC group $(p<$ 
TABLE 1: Comparison of outcome measures in an obese human study.

\begin{tabular}{lccc}
\hline & Before & After & $p$ value \\
\hline BW (kg) & $65.37 \pm 3.53$ & $64.10 \pm 3.49$ & 0.02 \\
BMI & $24.93 \pm 0.65$ & $24.28 \pm 0.58$ & 0.047 \\
Waist-hip ratio & $0.90 \pm 0.01$ & $0.90 \pm 0.01$ & 0.79 \\
Percent body fat (\%) & $29.71 \pm 1.44$ & $29.56 \pm 1.39$ & 0.58 \\
BUN (mg/dl) & $15.28 \pm 1.32$ & $14.52 \pm 1.14$ & 0.44 \\
Creatinine (mg/dl) & $0.72 \pm 0.39$ & $0.72 \pm 0.42$ & 0.75 \\
eGFR & $97.38 \pm 6.71$ & $93.58 \pm 4.94$ & 0.36 \\
AST (U/L) & $32.42 \pm 6.96$ & $27.04 \pm 2.53$ & 0.51 \\
ALT (U/L) & $35.25 \pm 13.56$ & $25.96 \pm 5.40$ & 0.28 \\
GGT (U/L) & $44.91 \pm 13.53$ & $43.04 \pm 13.49$ & 0.77 \\
Protein (g/dl) & $6.98 \pm 0.53$ & $6.79 \pm 0.55$ & 0.21 \\
Albumin (g/dl) & $3.99 \pm 0.32$ & $3.98 \pm 0.48$ & 0.97 \\
Fasting glucose (mg/dl) & $94.5 \pm 5.6$ & $88.3 \pm 9.99$ & 0.12 \\
HbAlc (\%) & $6.11 \pm 0.58$ & $5.97 \pm 0.56$ & 0.28 \\
\hline
\end{tabular}

"Before" and "after" indicate before Euphorbia administration and after Euphorbia administration, respectively. Data are presented as mean \pm $\mathrm{SE}$. For $p$ value, the results are from paired $t$-test to examine withingroup effects. BW: body weight; BMI: body mass index; BUN: blood urea nitrogen; eGFR: CockCroft-Gault estimated glomerular filtration rate; AST: aspartic aminotransferase; ALT: alanine aminotransferase; GGT: gamma glutamyltranspeptidase.

0.001) and was significantly lower in the Euphorbia group compared to the HFD group $(p<0.05)$. Insulin resistance calculated by HOMA-IR was significantly higher in the HFD group than NC group $(p<0.05)$, while the HOMA-IR of the Euphorbia group was significantly lower than that HFD group $(p<0.01)$ (Figure 1(a)).

OGTT, conducted at 12 weeks after initiation of the study, showed the highest blood glucose concentration at 30 minutes in every group and decreased gradually. The 30-, 60-, and 120-minute glucose levels of the HFD group were elevated significantly compared to those of the NC group $(p<0.05)$ and were significantly lower in the Euphorbia group than in the HFD group $(p<0.05)$. The area under the curve (AUC) also showed similar tendencies, with the AUC of the HFD group being higher than that of the NC group, while that of the Euphorbia group was significantly lower than that of the HFD group $(p<0.01)$ (Figure $1(b)$ ).

3.4. Effects on Expression of CD68, TNF- $\alpha, I F N-\gamma$, and IL-6 Genes. The gene expression of CD68 in liver was significantly higher in the HFD group than NC group $(p<0.001)$ and was significantly lower in the Euphorbia group compared to the HFD group $(p<0.01)$. And TNF- $\alpha$ in liver and epididymal fat was significantly higher in the HFD group than NC group ( $p<0.001$ ), and Euphorbia significantly lowers TNF- $\alpha$ gene expression compared to the HFD group $(p<0.001)$. IL-6 expression in epididymal fat was also higher in the HFD group than in the NC group and decreased significantly in the Euphorbia group compared to the HFD group $(p<0.05)$. The IFN- $\gamma$ in epididymal fat expression was significantly increased in the HFD group compared to the NC and Euphorbia groups $(p<0.05)$ (Figure $1(c))$.

3.5. Effects on Macrophages in Liver and Adipose Tissue. The F4/80 positive Kupffer cells and fat deposits were markedly increased in HFD (F4/80-stained surface of $4.61 \pm 0.64 \%$ in HFD versus $0.89 \pm 0.18 \%$ in NC, $p<0.01)$, and the number of Kupffer cells and lipid accumulation were decreased in Euphorbia group (2.20 $\pm 0.22 \%$ in Euphorbia versus HFD, $p<0.05$ ) (Figure 3 ). The RT-PCR analysis confirmed the markedly reduced the expression of CD68 mRNA in liver (Figure 1(c)). CD68 positive ATMs infiltration rate was significantly higher in the HFD group compared to the NC group ( $p<0.001)$, and Euphorbia showed significant decrease in ATMs percentage $(p<0.05)$ (Figure $2(\mathrm{a})$ ). The percentage of CD11c+ ATMs was significantly higher in the HFD group compared to the NC group $(p<0.05)$ and lower in the Euphorbia group compared to the HFD group, but the difference was not significant. In contrast, the CD206+ ATMs percentage was significantly lower in the HFD group than the NC group $(p<0.01)$ and significantly higher in the Euphorbia group than the HFD group $(p<0.01)$ (Figure 2(b)).

3.6. Immunofluorescence Staining of SVCs. Compared to the NC group, the HFD group showed an increase in the number of stained macrophages and lipid accumulation. For the Euphorbia group, the number of macrophages and lipid accumulation were decreased (Figure 3).

\section{Discussion}

In this study, we evaluated Euphorbia as a potential therapy for obesity and insulin resistance. We were able to obtain data on efficacy and safety of Euphorbia from human studies, since Euphorbia has already been approved for use as herbal medicine in the Republic of Korea when used by a professional physician [18]. Additionally, we conducted an in vivo study to estimate the efficacy, safety, and mechanism of Euphorbia, especially with respect to the anti-inflammatory effect.

In a study of 14 obese patients, we found that a 1-day prescription of $6 \mathrm{~g}$ Euphorbia caused significant decrease in BW and BMI. Because Euphorbia is a cathartic herbal medicine, the short-term effect of Euphorbia might mostly result from dehydration. However, in our human study, the weight loss effects persisted for one month, which allowed enough time to recover from dehydration. This result implies that the effect of Euphorbia depends on more than simple dehydration induced by diarrhea.

It was reported that administration of Euphorbia with herbal decoction to obese rats induced weight loss [17]. In agreement, administration of Euphorbia in the present study induced significant decrease in BW and epididymal fat weight, which is equivalent to visceral fat in humans. A decrease in epididymal fat weight after a five-week administration of Euphorbia in mice demonstrated the antiobesity effect of Euphorbia. In a previous study [16] of ethanol 

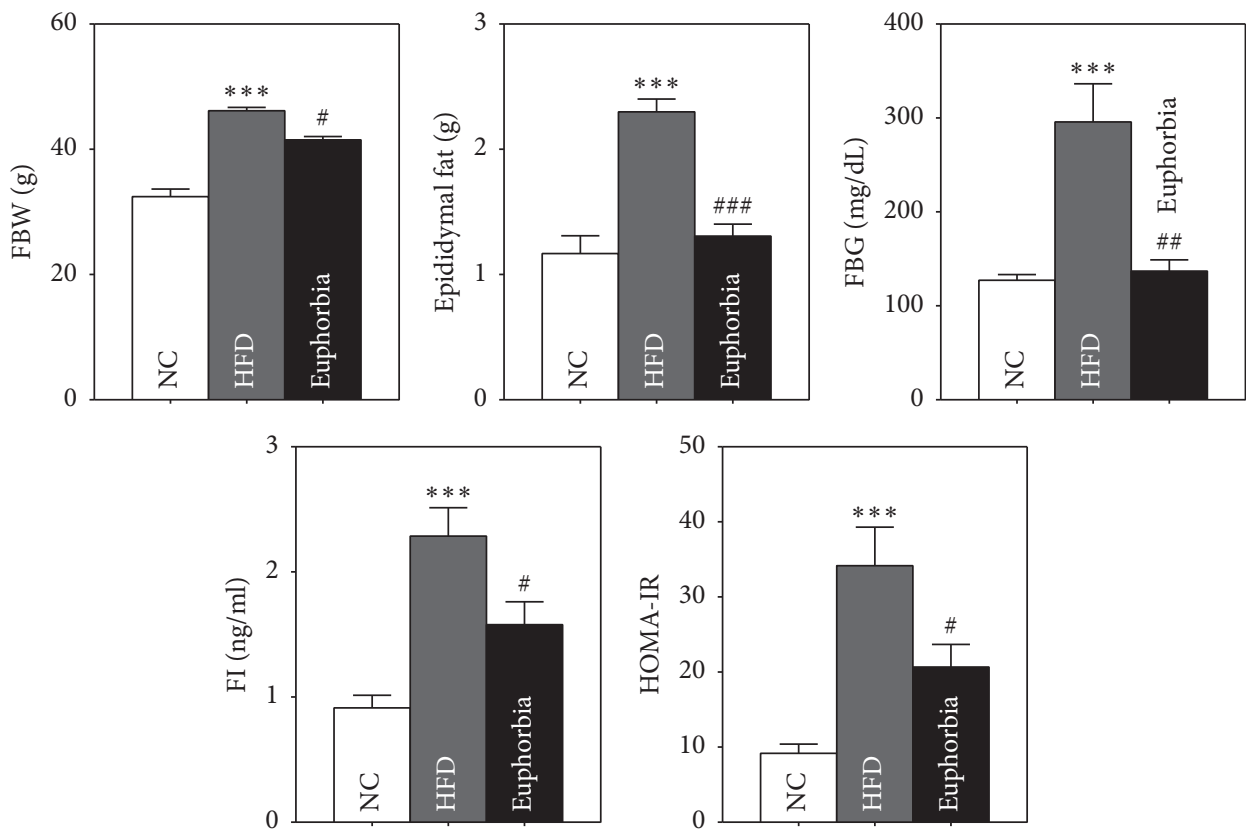

(a)
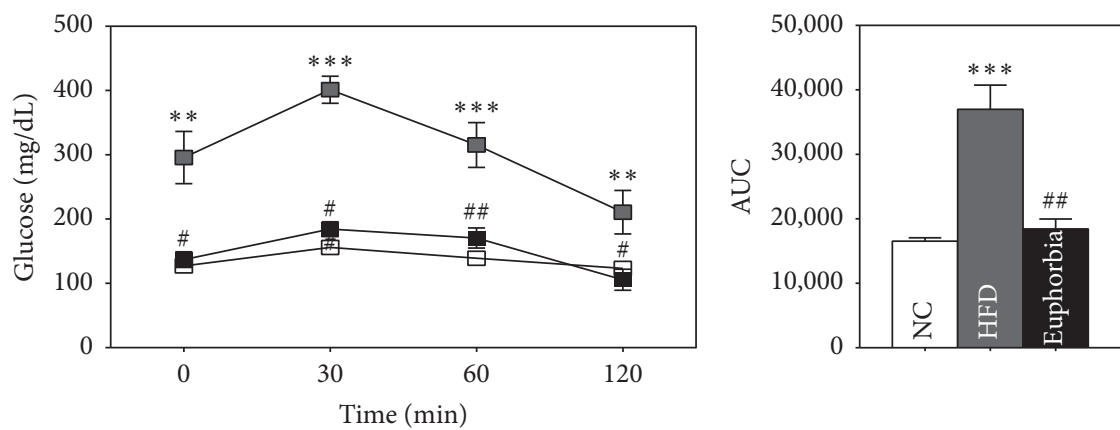

$$
\begin{aligned}
& \square \text { NC } \\
& -\square-\text { HFD } \\
& - \text { - Euphorbia }
\end{aligned}
$$

(b)
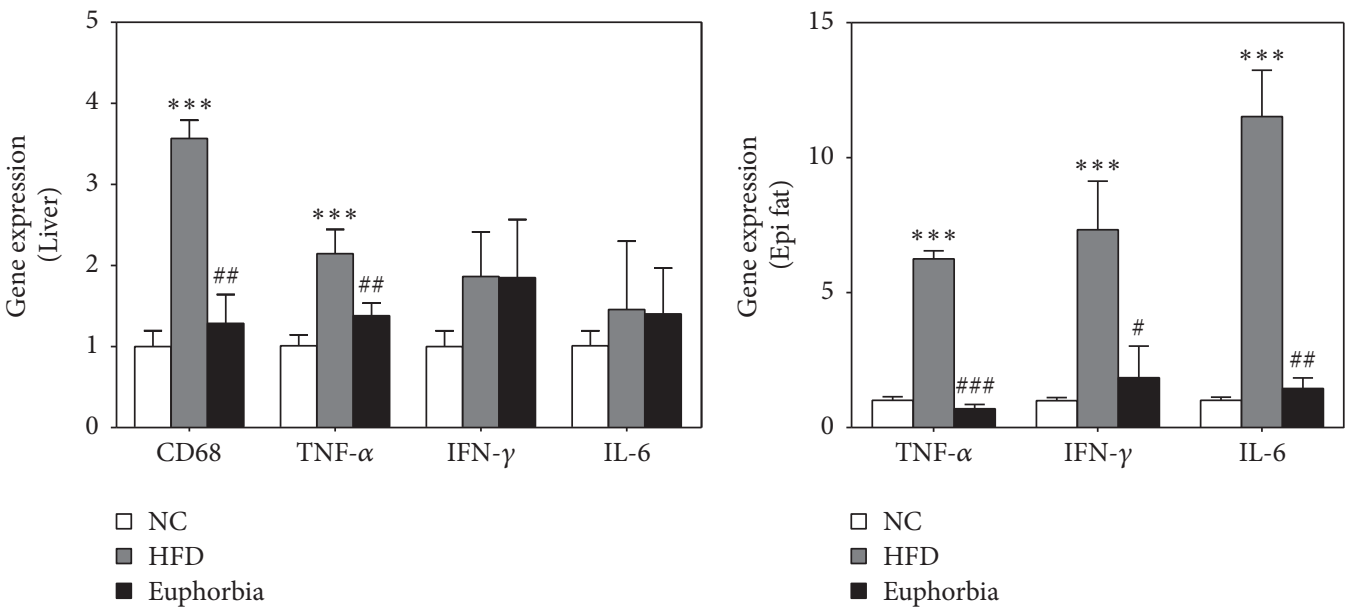

(c)

FIGURE 1: Main results of the in vivo study. (a) Fasting body weight (FBW), epididymal fat weight, fasting blood glucose (FBG), fasting insulin (FI), HOMA-IR of normal chow NC, HFD, and Euphorbia groups. (b) Oral glucose tolerance test (OGTT) and area under curve (AUC) of the three groups. (c) Expression of CD68, TNF- $\alpha$, IFN- $\gamma$, and IL-6 genes in the three groups. ${ }^{* *} p<0.01$ and ${ }^{* * *} p<0.001$, HFD compared with NC; ${ }^{\#} p<0.05,{ }^{\# \#} p<0.01$, and ${ }^{\# \# \#} p<0.001$, Euphorbia compared with HFD. NC: NC group; HFD: HFD group; Euphorbia: Euphorbia group. 

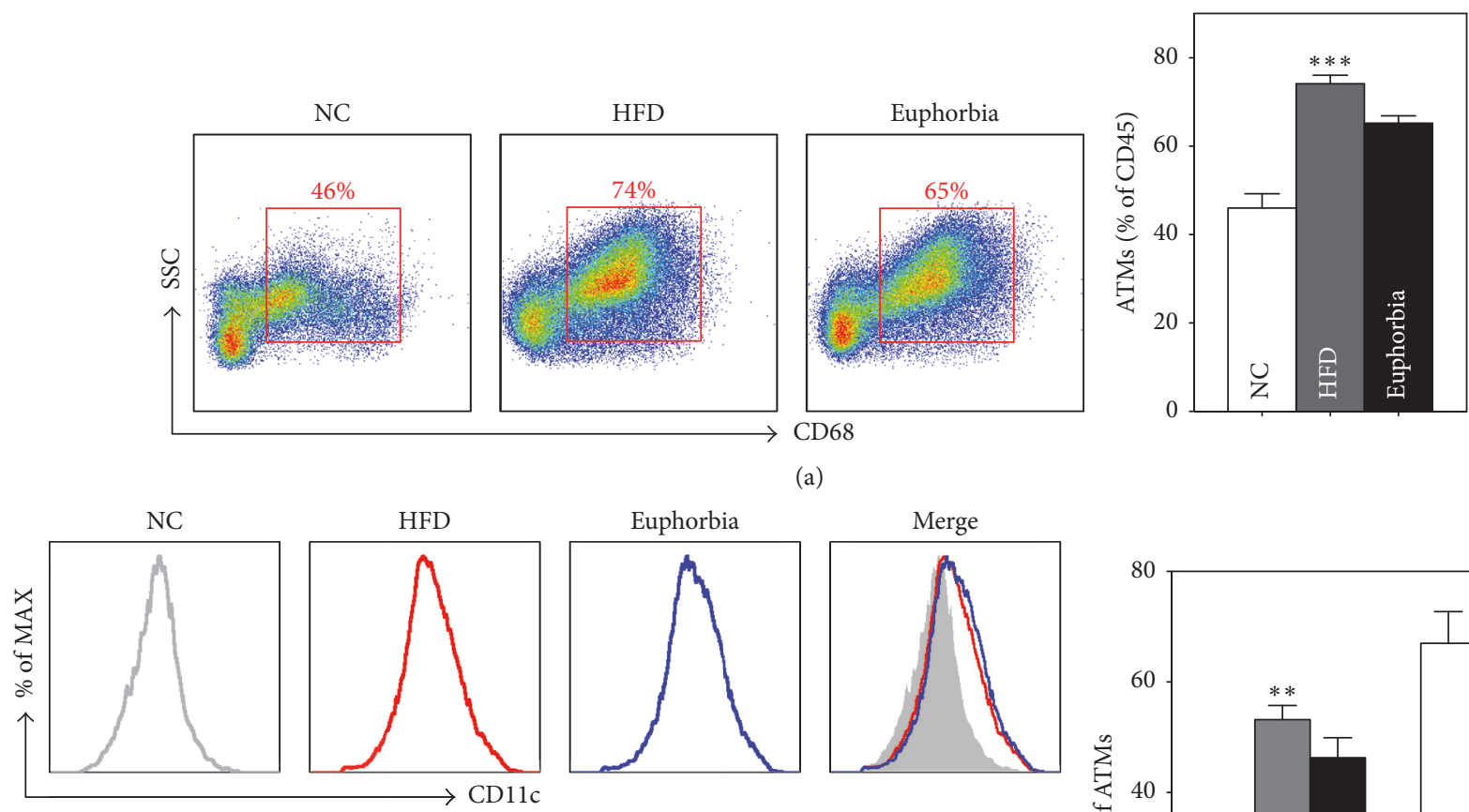

(a)
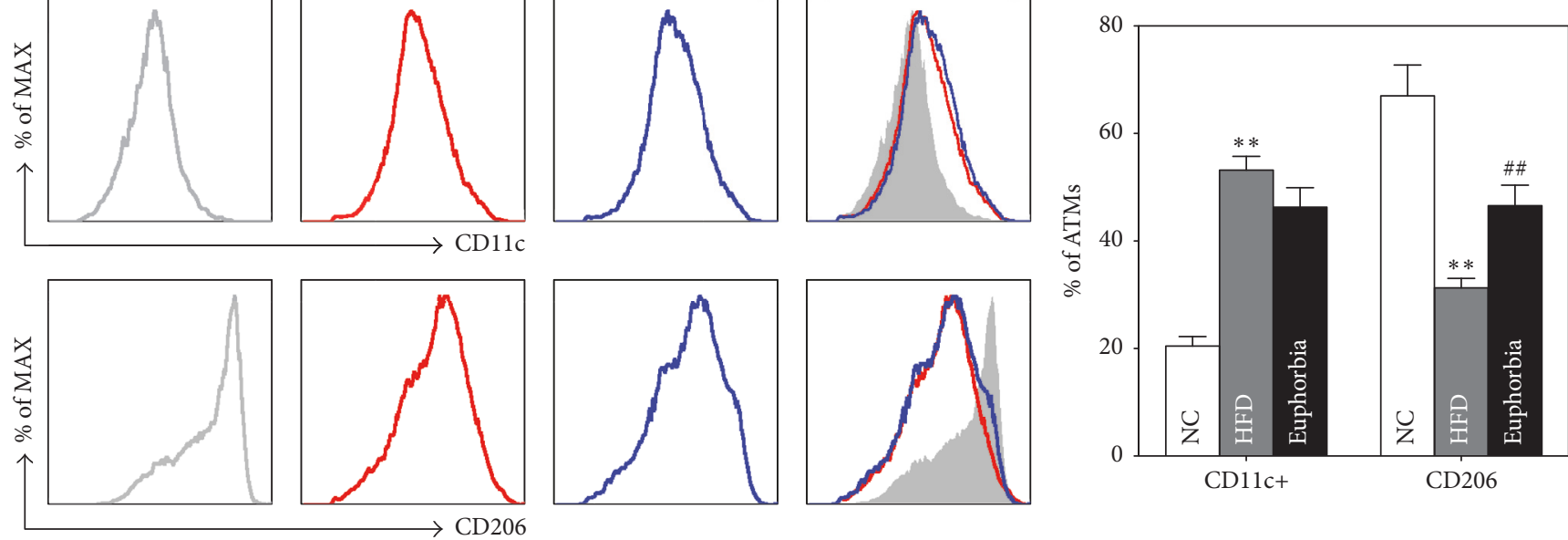

- NC
- HFD
- Euphorbia

(b)

FIgURE 2: (a) ATM infiltration rate; (b) CD206- and CD1lc-positive ATMs. NC: NC group; HFD: HFD group; Euphorbia: Euphorbia group. ${ }^{* *} p<0.01 ;{ }^{* * *} p<0.001$ versus NC group. ${ }^{\# \#} p<0.01$ versus HFD group.

extracts of Euphorbia, a new tetracyclic triterpenoid of Euphorbia and five known triterpenoids with euphane skeletons were shown to be potentially useful in the clinical treatment of diabetes. In this study, Euphorbia showed antihyperglycemic effects on FBG and OGTT of obese mice. In addition, changes in FI and HOMA-IR indicate that Euphorbia improves IR.

It has been shown that high-fat diet increases Kupffer cells number and induces their proinflammatory status, and cytokine and chemokine production by activated Kupffer cells is involved in the pathogenesis of liver damage [19]. Moreover, visceral adipose tissue is an endocrine organ that releases adipokines and cytokines, which have a role in fat metabolism and insulin sensitivity [20]. Particularly, macrophage accumulation with increased cytokines was observed in adipose tissue, which implies the chronic inflammatory features of obesity $[21,22]$. In this study, Euphorbia administration was related to a significant decrease in gene expression of cytokines TNF- $\alpha$, IFN- $\gamma$, and IL-6. TNF- $\alpha$ is known to reduce insulin sensitivity by impairing insulin signal transduction [23]. Also, an increase in the expression of IFN- $\gamma$ and IL- 6 was also associated with insulin resistance $[24,25]$.

The rate of Kupffer cells in liver and ATM infiltration into adipose tissue was also decreased in the Euphorbia-treated group. Particularly, the percentage of CD206+ ATMs, which indicates the anti-inflammatory activity of ATMs in adipose tissue, was significantly increased in the Euphorbia-treated group in comparison to the HFD group [26]. This result suggests that improvement of hyperglycemia and IR in the Euphorbia group is correlated with the anti-inflammatory effects of Euphorbia on liver and visceral adipose tissue.

In obesity, adipocyte-hypertrophy leads to hypoxia, endoplasmic reticulum stress, and lipotoxicity, which result in increased macrophage infiltration accompanied by elevated concentrations of inflammatory markers. The accumulation of macrophages in adipose tissue leads to a specific feature called crown-like structure, which is the 

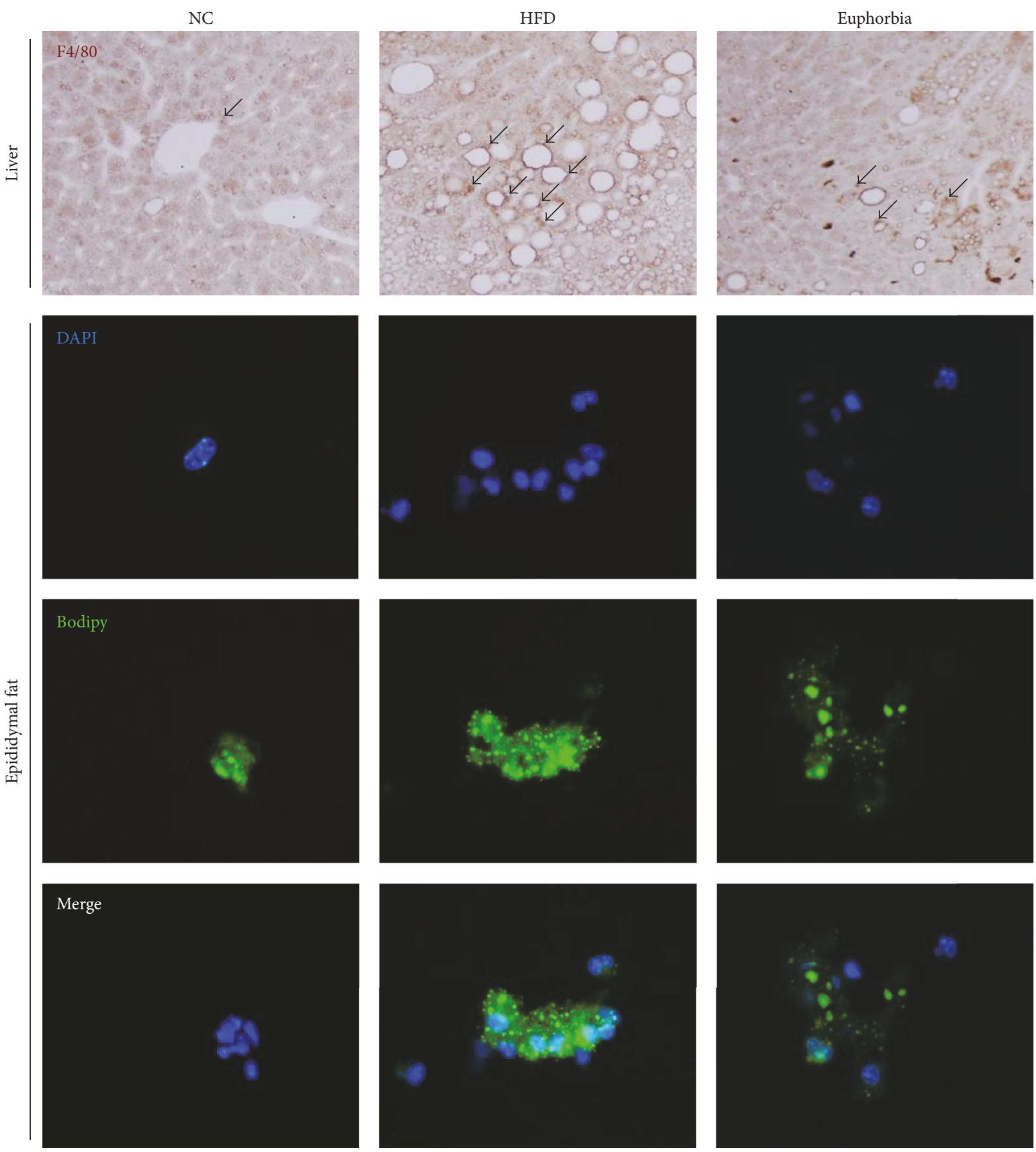

FIGURE 3: Immunohistochemical staining (F4/80) of liver and immunofluorescence staining of stromal vascular cells. Arrow indicates F4/80 positive Kupffer cells. NC: NC group; HFD: HFD group; Euphorbia: Euphorbia group.

cluster of macrophages around dead adipocytes [27]. We obtained SVCs from the epididymal fat pad and analyzed them using immunofluorescence microscopy. The SVCs from the Euphorbia group showed a decreased number of macrophages with a smaller amount of lipid accumulation compared to those in the HFD group.

Taken together, the degree of lipid accumulation in liver and visceral fat, Kupffer cells, ATM infiltration rate, which produces proinflammatory cytokines in liver and adipose tissue, and gene expression of cytokines are important for evaluating antiobesity, anti-inflammatory, and anti-IR effects. In this study, the BW decrease caused by Euphorbia treatment persisted for 1 month in humans, and the in vivo studies showed epididymal fat weight decrease, decline in Kupffer cells and ATM percentage, and decreased expression of TNF- $\alpha$, IFN- $\gamma$, and IL- 6 genes. These results indicate that Euphorbia has an antiobesity effect, which consequently induces an anti-inflammatory effect that improves IR. 


\section{Conclusion}

In conclusion, our study showed that Euphorbia improves body weight and insulin resistance in human and in vivo study. A possible mechanism to explain this result is the reduction in the inflammatory reaction due to decreased Kupffer cells and adipose tissue macrophage infiltration. The cathartic effects of Euphorbia necessitate further research to assess issues with diarrhea that might affect long-term use, even though the results suggest that Euphorbia can be used to effectively improve obesity-related IR.

\section{Disclosure}

The authors are responsible for the writing and content of the paper.

\section{Conflicts of Interest}

The authors have no conflicts of interest.

\section{Authors' Contributions}

Seung-Wook Lee and Hyun-Young Na contributed equally to this work.

\section{Acknowledgments}

This study was supported by the Traditional Korean Medicine Research and Development Program funded by the Ministry of Health and Welfare through Korea Health Industry Development Institute (Grant no. HI13C0700).

\section{References}

[1] C. L. Ogden, M. D. Carroll, and K. M. Flegal, "Prevalence of obesity in the United States: Reply," JAMA - Journal of the American Medical Association, vol. 312, no. 2, pp. 189-190, 2014.

[2] A. R. Aroor, S. McKarns, V. G. Demarco, G. Jia, and J. R. Sowers, "Maladaptive immune and inflammatory pathways lead to cardiovascular insulin resistance," Metabolism: Clinical and Experimental, vol. 62, no. 11, pp. 1543-1552, 2013.

[3] Z. Yang and N. Zhang, "The burden of overweight and obesity on long-term care and medicaid financing," Medical Care, vol. 52, no. 7, pp. 658-663, 2014.

[4] H. R. Wyatt, "Update on treatment strategies for obesity," Journal of Clinical Endocrinology and Metabolism, vol. 98, no. 4, pp. 1299-1306, 2013.

[5] R. Padwal, S. K. Li, and D. C. Lau, "Long-term pharmacotherapy for obesity and overweight." Cochrane database of systematic reviews (Online), no. 3, p. CD004094, 2004.

[6] T. M. Ehrman, D. J. Barlow, and P. J. Hylands, "Phytochemical informatics of traditional Chinese medicine and therapeutic relevance," Journal of Chemical Information and Modeling, vol. 47, no. 6, pp. 2316-2334, 2007.

[7] C. G. Zhao ZX, S. Xie, XL. Zhou, and XL. Hu, "Dressing navel with Euphorbia kansui in combination with taken DuShenTang orally to cure ascites caused by hepatocirrhosis in clinic," Journal of Emergency in Traditional Chinese Medicine, vol. 19, pp. 388-389.
[8] Y. Zhang, X. S. Lv, Y. X. Li, H. H. Tang, X. R. Li, and F. Y. Wu, "Therapeutic effect of kansui root on patients with severe acute pancreatitis," Chinese Journal of General Surgery, vol. 13, pp. 401404, 2004.

[9] D. M. Ouyang JB and YM. Ouyang, "Therapeutic effect on adjuvant treatment of severe acute pancreatitis with Euphorbia kansui Lious," China Journal of Modern Medicine, vol. 14, 2004.

[10] Y. C. D. Fan, X. X. Gu, G. Y. Wang, and Ma J., "Treatment of intestinal obstruction with a large dose of kansui," Journal of Emergency in Traditional Chinese Medicine, vol. 14, pp. 278-279, 2005.

[11] L. C. Whelan and M. F. Ryan, "Ethanolic extracts of Euphorbia and other ethnobotanical species as inhibitors of human tumour cell growth," Phytomedicine, vol. 10, no. 1, pp. 53-58, 2003.

[12] Z. WF, "Study on in vivo antiviral activity of four diterpenoids from ethanol extracts of Euphorbia kansui," Chinese Traditional Herbal Drugs, vol. 35, pp. 65-68, 2004.

[13] W. F. Zheng, Z. Cui, and Q. Zhu, "Cytotoxicity and antiviral activity of the compounds from Euphorbia kansui," Planta Medica, vol. 64, no. 8, pp. 754-756, 1998.

[14] S. Nunomura, S. Kitanaka, and C. Ra, "3-O-(2,3dimethylbutanoyl)-13-odecanoylingenol from Euphorbia kansui suppresses IgE-mediated mast cell activation," Biological and Pharmaceutical Bulletin, vol. 29, no. 2, pp. 286-290, 2006.

[15] R. C. Dutra, P. R. De Cezaro De Souza, A. F. Bento et al., "Euphol prevents experimental autoimmune encephalomyelitis in mice: Evidence for the underlying mechanisms," Biochemical Pharmacology, vol. 83, no. 4, pp. 531-542, 2012.

[16] J. Guo, L.-Y. Zhou, H.-P. He, Y. Leng, Z. Yang, and X.-J. Hao, "Inhibition of $11 \beta$-HSD1 by tetracyclic triterpenoids from euphorbia kansui," Molecules, vol. 17, no. 10, pp. 11826-11838, 2012.

[17] J.-W. Kim and E.-Y. Kim, "The effect of HyungbangdojucksanGami and Kamsuchunilhwan on the obesity in the Rats," Journal of Sasang Constitutional Medicine, vol. 12, pp. 184-194, 2000.

[18] Ministry of Food and Drug Safety, The Korean Herbal Pharmacopoeia, Shinil Books, Seoul, South Korea, 2011.

[19] M. Sharma, S. Mitnala, R. K. Vishnubhotla, R. Mukherjee, D. N. Reddy, and P. N. Rao, "The riddle of nonalcoholic fatty liver disease: progression from nonalcoholic fatty liver to nonalcoholic steatohepatitis," Journal of Clinical and Experimental Hepatology, vol. 5, no. 2, pp. 147-158, 2015.

[20] P. E. Scherer, "Adipose tissue: from lipid storage compartment to endocrine organ," Diabetes, vol. 55, no. 6, pp. 1537-1545, 2006.

[21] S. P. Weisberg, D. McCann, M. Desai, M. Rosenbaum, R. L. Leibel, and A. W. Ferrante Jr., "Obesity is associated with macrophage accumulation in adipose tissue," Journal of Clinical Investigation, vol. 112, no. 12, pp. 1796-1808, 2003.

[22] H. Xu, G. T. Barnes, Q. Yang et al., "Chronic inflammation in fat plays a crucial role in the development of obesity-related insulin resistance," The Journal of Clinical Investigation, vol. 112, no. 12, pp. 1821-1830, 2003.

[23] G. Kollias and P. Sfikakis, TNF Pathophysiology, vol. 11, KARGER, Basel, 2010.

[24] N. Paquot, M. J. Castillo, P. J. Lefèbvre, and A. J. Scheen, "No increased insulin sensitivity after a single intravenous administration of a recombinant human tumor necrosis factor receptor: fc fusion protein in obese insulin-resistant patients," The Journal of Clinical Endocrinology \& Metabolism, vol. 85, no. 3, pp. 1316-1319, 2000. 
[25] R. W. O’Rourke, M. D. Metcalf, A. E. White et al., "Depotspecific differences in inflammatory mediators and a role for NK cells and IFN- $\gamma$ in inflammation in human adipose tissue," International Journal of Obesity, vol. 33, no. 9, pp. 978-990, 2009.

[26] B.-C. Lee and J. Lee, "Cellular and molecular players in adipose tissue inflammation in the development of obesity-induced insulin resistance," Biochimica et Biophysica Acta-Molecular Basis of Disease, vol. 1842, no. 3, pp. 446-462, 2014.

[27] J. L. Schultze, A. Schmieder, and S. Goerdt, "Macrophage activation in human diseases," Seminars in Immunology, vol. 27, no. 4, pp. 249-256, 2015. 


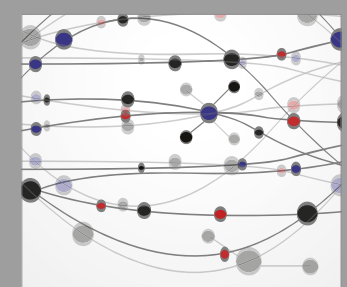

The Scientific World Journal
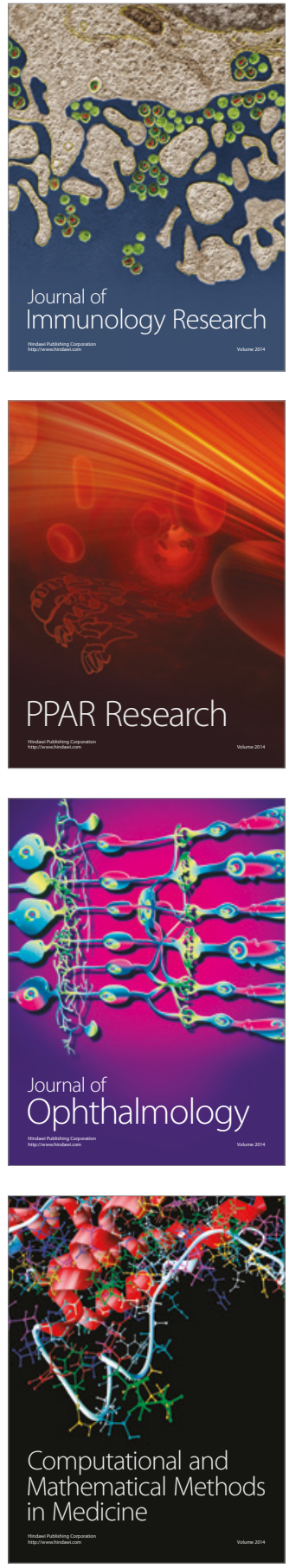

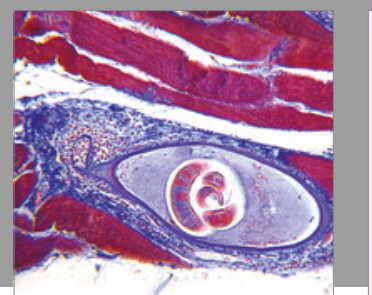

Gastroenterology Research and Practice
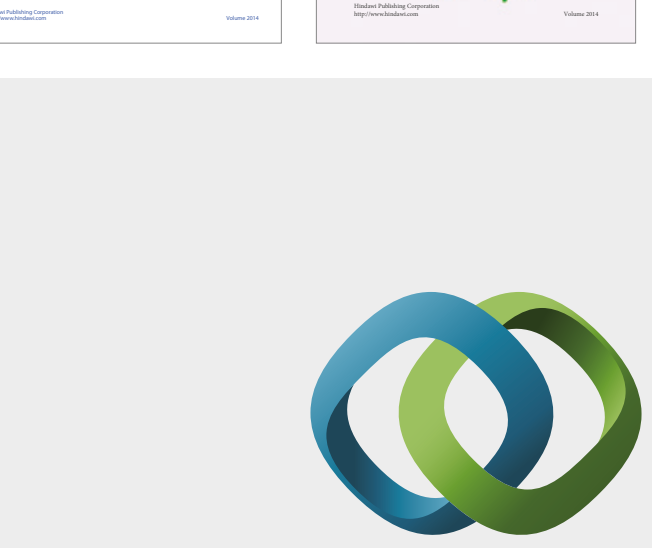

\section{Hindawi}

Submit your manuscripts at

https://www.hindawi.com
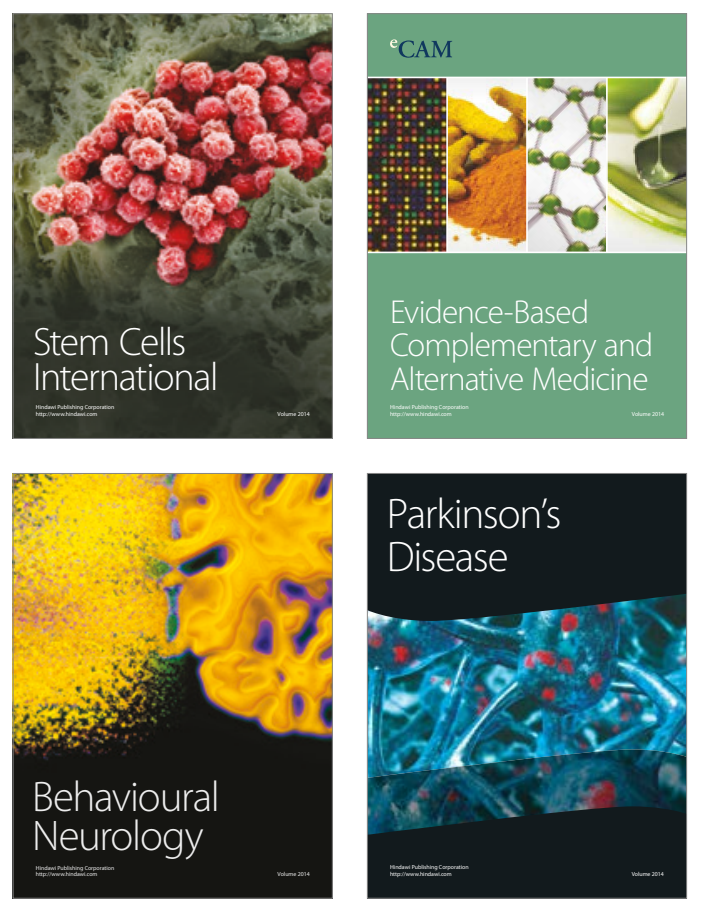
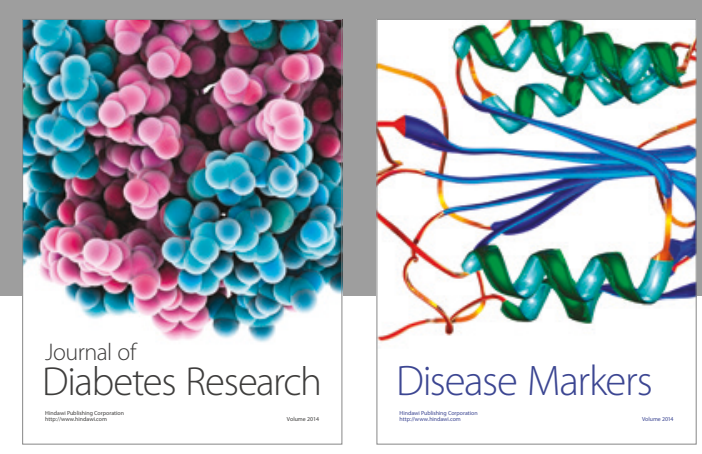

Disease Markers
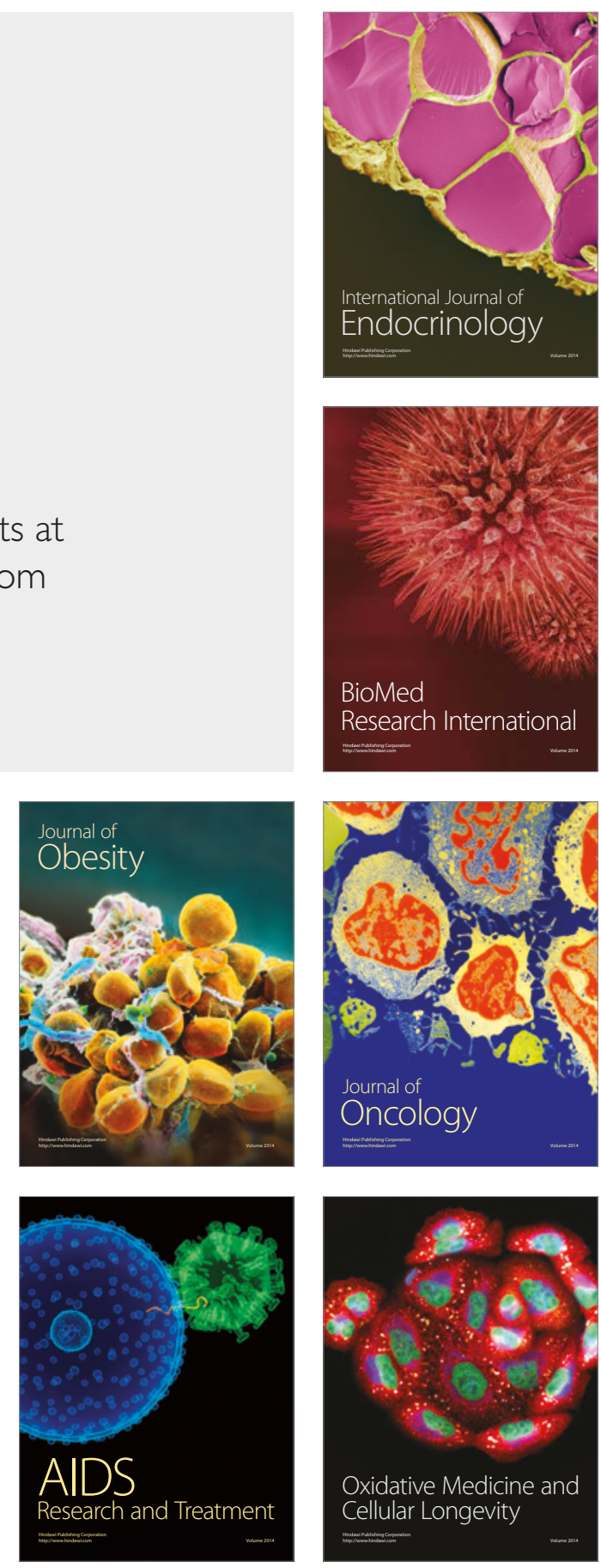